The Society shall not be responsible for statements or opinions advanced in papers or in dis. cussion at meetings of the Society or of its Divisions or Sections, or printed in its publications. Discussion is printed only if the paper is published in an ASME Journal. Papers are available from ASME for fifteen months after the meeting.

Printed in USA.

\title{
Rotor-Stator Interaction in a Diffuser Pump
}

\author{
N. ARNDT, Graduate Student \\ A. J. ACOSTA, Professor \\ C. E. BRENNEN, Professor \\ T. K. CAUGHEY, Professor \\ California Institute of Technology \\ Pasadena, CA 91125 USA
}

\section{ABSTRACT}

The interaction between impeller blades and diffuser vanes in a diffuser pump was investigated. Steady and unsteady pressure measurements were taken on the diffuser vanes, and the shroud wall of a vaned and a vaneless diffuser. Steady, unsteady, and ensemble averaged unsteady data, as well as frequency spectra are presented. The measurements were made for different flow coefficients, shaft speeds, and radial gaps between impeller blade trailing and diffuser vane leading edge $(1.5 \%$ and $4.5 \%$ based on impeller discharge radius). The resulting lift on the vane, both steady and unsteady, was computed from the pressure measurements at mid vane height. The magnitude of the fluctuating lift was found to be greater than the steady lift. The pressure fluctuations were larger on the suction side than on the pressure side attaining their maximum value, of the same order of magnitude as the total pressure rise across the pump, near the leading edge. Pressure fluctuations were also measured across the span of the vane, and those near the shroud were significantly smaller than those near the hub. The pressure fluctuations on the shroud wall itself were larger for the vaned diffuser than a vaneless diffuser. Lift, vane pressure, and shroud wall pressure fluctuations decreased strongly with increasing radial gap.

\section{INTRODUCTION}

Blade and vane design in centrifugal pumps is currently based on the assumption that the flow in both impeller and diffuser is steady. This however implies that the radial gap between impeller discharge and diffuser inlet is large so that no flow unsteadiness of any kind due to blade row interaction will occur. If, however, the rows are closely spaced there may be a strong interaction which may influence both the aerodynamic and structural performance of blades and vanes. In some cases, this has led to vane failure. There are few practical analytical or numerical methods or experimental results available to provide knowledge about the magnitude of this unsteadiness.

The impeller-diffuser interaction may be divided into two different mechanisms; potential flow interaction and wake interaction. In general, wake interaction extends further downstream, but if impeller and diffuser are closely spaced, both mechanisms will occur simultaneously. Most of the experimental work on blade row interaction with the aim of measuring unsteady blade pressures has been done in axial turbomachines. Among others, Dring [1] investigated blade row interaction in an axial turbine, and found both potential flow and wake interaction for closely spaced blade rows ( $15 \%$ based on chord). Gallus [2],[3] reported measurements on centrifugal compressors. The blade rows were spaced relatively far apart ( $60 \%$ based on chord), such that the potential interaction between rotor and stator was weak. In radial turbomachinery, impeller blade pressure measurements were reported by lino [4] and flow field investigation in centrifugal impellers have been made among others by Krain [5], and Cumptsy and Inoue [6].

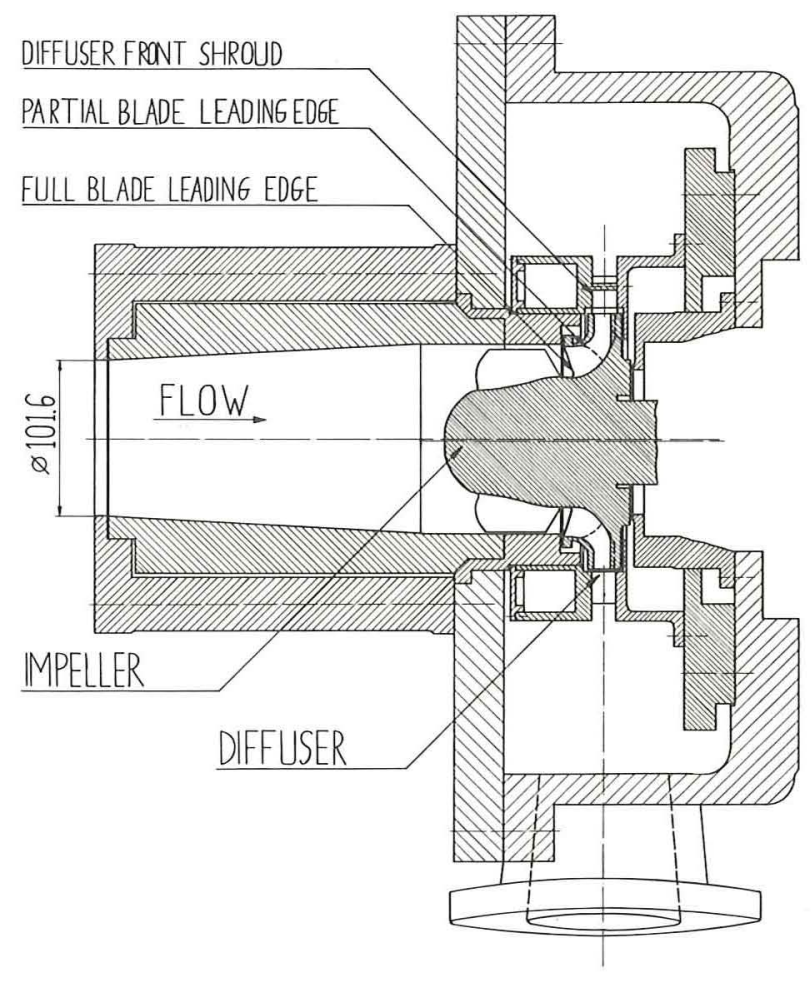

Fig. 1 Simplified view of the test section. 


\section{NOMENCLATURE}

$A_{2}$

impeller discharge area

$c \quad$ vane chord

$c_{f} \quad$ front shroud pressure coefficient, $c_{f}=p_{f} /(1 / 2) \rho u_{2}^{2}$

$c_{i} \quad$ magnitude of $i$ 'th Fourier coefficient, normalized by $(1 / 2) \rho u_{2}^{2}$

$c_{L} \quad$ lift coefficient, $c_{L}=L /(1 / 2) \rho u_{2}^{2} c$

$\bar{c}_{p} \quad$ steady vane pressure coefficient, $\bar{c}_{p}=\left(\bar{p}_{v}-\bar{p}_{u p}\right) /(1 / 2) \rho u_{2}^{2}$

$\tilde{c}_{p} \quad$ unsteady vane pressure coefficient, $\tilde{c}_{p}=\tilde{p}_{v} /(1 / 2) \rho u_{2}^{2}$

$\tilde{c}_{p, a v} \quad$ ensemble averaged unsteady vane pressure coefficient, $\tilde{c}_{p, a v}=\tilde{p}_{v, a v} /(1 / 2) \rho u_{2}^{2}$

$c_{p, a v} \quad$ ensemble averaged vane pressure coefficient, $c_{p, a v}=\bar{c}_{p}+\tilde{c}_{p, a v}$

frequency

impeller blade passage frequency, $f_{b}=z_{b}(\mathrm{rpm} / 60)$

lift (= component of the force vector on the vane normal to the chord joining the vane leading and the vane trailing edge)

outward normal vector on diffuser vane

pressure

total pressure

flow rate

radius

impeller discharge radius $\left(R_{2}=83.82 \mathrm{~mm}\right)$

diffuser inlet radius

revolutions per minute

non-dimensionalized arclength on vane suction side

impeller tip speed, $u_{2}=2 \pi R_{2}(\mathrm{rpm} / 60)$

diffuser vane coordinates

number of impeller blades, $z_{b}=8$

number of full impeller blades $\left(z_{f b}=4\right)$, number of partial impeller blades $\left(z_{p b}=4\right)$

parametric diffuser vane coordinate

density

flow coefficient, $\phi=Q / u_{2} A_{2}$

total head coefficient, $\psi=\left(p_{\text {down }}-p_{\text {up }}\right) / \rho u_{2}^{2}$
Herein, results on the interaction between a centrifugal impeller; namely one half of the double suction pump of the HPOTP (High Pressure Oxygen Turbopump) of the SSME (Space Shuttle Main Engine) and a vaned diffuser will be reported. Data will be presented on steady and unsteady vane pressure measurements along mid vane height as well as at different axial positions across the vane. Superimposing the steady and ensemble averaged unsteady vane pressure, the ensemble averaged vane pressure was obtained (it was assumed that the steady pressure value, measured with mercury manometers, was identical to the time mean pressure value about which the piezoelectric pressure transducers, used for the unsteady measurements, measured the unsteady pressure). Steady and unsteady computations of the force on the vane were made from the pressure measurements. The lift on the vane was defined as the force component normal to the chord joining the vane leading and trailing edge. Surface pressure measurements were also made at the front shroud of the vaned and a vaneless diffuser.

During the tests, the impeller could only be positioned on locations on an orbit concentric to the diffuser center (orbit radius $=1.27 \mathrm{~mm}$ ), so that the radial gap between impeller blade trailing edge and the leading edge of any particular instrumented diffuser vane could be varied between $1.5 \%$ and $4.5 \%$, based on impeller discharge radius. The experiments were performed for radial gaps between impeller blade trailing edge and diffuser vane leading edge of $1.5 \%$ and $4.5 \%$. The flow past a diffuser vane is steady in the mean; for this reason we believe the pressure fluctuations observed there are due primarily to the local interaction between the impeller blades and the diffuser vane, and not to the proximity of neighboring diffuser vanes with a slightly different radial gap. Some preliminary results of this work have been previously reported in [7].

\section{TEST FACILITY AND INSTRUMENTATION}

The experiments were conducted in a recirculating water test loop. A simplified view of the test section is shown in figure 1 . One side of the double suction pump impeller of the HPOTP of the SSME was used for the tests (figure 2). The impeller has eight blades in total with four partial blades, and an inducer with four inducer blades. In order to fit into the test facility, the impeller discharge diameter had to be reduced from $172.7 \mathrm{~mm}$ to $167.64 \mathrm{~mm}$ The blade angle of both full and partial blades at impeller discharge is about 36 degrees.

\section{Subscripts}

$\begin{array}{ll}a v & \text { ensemble averaged } \\ b & \text { impeller blade } \\ \text { down } & \text { downstream } \\ f & \text { front shroud } \\ f b, p b & \text { full impeller blade, partial impeller blade } \\ \max & \text { maximum } \\ u p & \text { upstream } \\ v & \text { diffuser vane }\end{array}$

\section{Superscripts}

$$
\begin{array}{ll}
- & \text { steady } \\
& \text { unsteady }
\end{array}
$$

\section{ABBREVIATIONS}

\section{FB full impeller blade}

FP pressure side of full impeller blade

FS suction side of full impeller blade

HPOTP High Pressure Oxygen Turbopump

$\mathrm{LE}$ diffuser vane leading edge

PCB PCB Piezoelectronics, INC. Depew, NY 14043

PB partial impeller blade

PP pressure side of partial impeller blade

PS suction side of partial impeller blade

PS diffuser vane pressure side

SS diffuser vane suction side

SSME Space Shuttle Main Engine

TE diffuser vane trailing edge

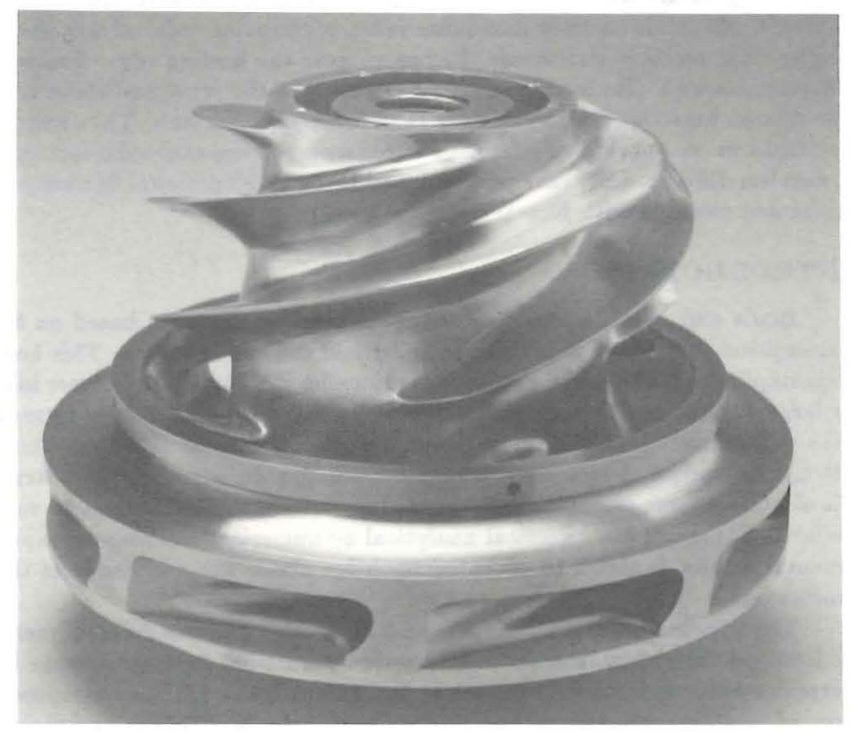

Fig. 2 Test impeller. 


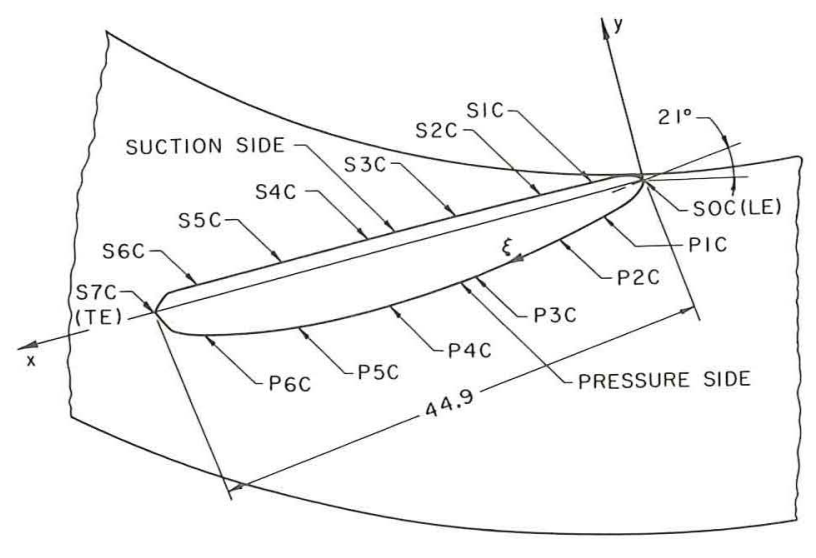

Fig. 3a Diffuser vane showing the location of the pressure taps at mid vane height.

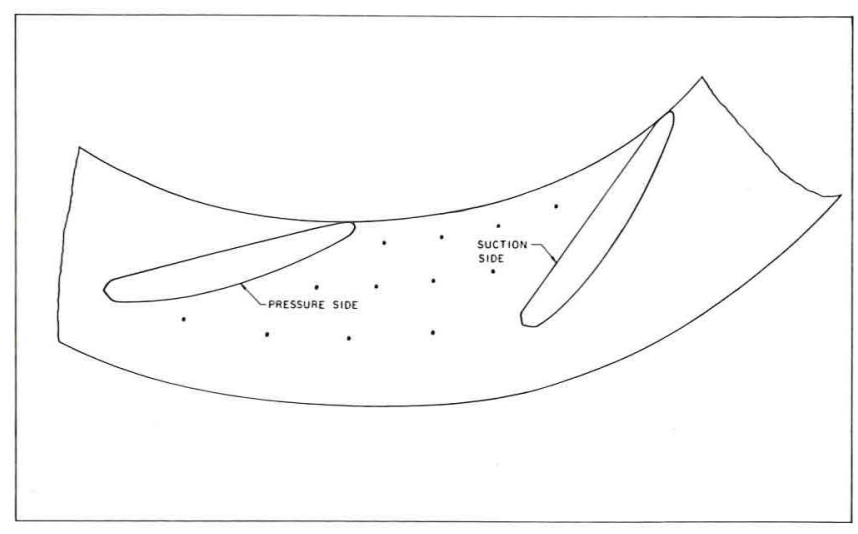

Fig. 3b Front shroud of Diffuser $S$ showing the location of the diffuser front shroud pressure measurements.

The vaned diffuser, referred to as Diffuser $\mathrm{S}$, is a straight wall constant width diffuser with nine vanes. No volute, however, is provided. Thus, the flow is discharged from the diffuser into a large housing. The shape of a vane, with the pressure taps at mid vane height, is shown in figure $3 \mathrm{a}$. It is identical to the one used in an early version of the diffuser of the HPOTP of the SSME; however, the number of vanes was reduced from seventeen in the diffuser of the SSME to nine in the experiment. Vane and front shroud pressure measurements were made on this diffuser. A second diffuser, referred to as Diffuser T, of identical geometry as Diffuser S but vaneless, was used for front shroud pressure measurements.

Diffuser vane pressure measurements were made at twenty different locations on the vanes, fourteen of those located at mid vane height of pressure and suction side (figure $3 \mathrm{a}$ ), the remaining six being distributed axially between front shroud and hub (or back shroud) at two different locations downstream of the leading edge on the suction side of the vane. The size of the pressure transducer did not permit arbitrarily close spacing of the pressure taps on a single vane, so that a total of seven vanes was used for the twenty pressure taps. Front shroud pressure measurements were made for Diffuser $\mathrm{S}$ and Diffuser $\mathrm{T}$ at three different distances from the diffuser inlet. For Diffuser S, at any of those three distances four measurements were taken at different circumferential positions between suction and pressure side of two adjacent vanes (figure $3 \mathrm{~b}$ ).
Steady vane pressure measurements were obtained by using conventional mercury manometers. As reference pressure, the wall pressure at the intake about $260 \mathrm{~mm}$ upstream of the inducer blade leading edge of the impeller was used. The experimental error on those measurements was estimated to be $\pm 0.5 \%$. For impeller back flow observations, tufts were placed at different locations upstream of the inducer blade leading edge. For the flow coefficients, on which steady vane pressure measurements are presented $(\phi=0.12$ and 0.09 ) and for which steady vane pressure measurement are used in the vane lift computations ( $\phi=0.15,0.12$ and 0.09$)$, the region of impeller back flow did at most extend to about $60 \mathrm{~mm}$ upstream of the inducer blade leading edge. Hence, the reference pressure was taken sufficiently far upstream of the impeller.

Piezoelectric pressure transducers from PCB Inc. were used for the unsteady measurements. The eigenfrequency (resonant frequency) of the transducer in air was given to be $300 \mathrm{kHz}$; therefore sufficiently high for the experiment. The linearity of the calibration, as provided by the manufacturer, was within $2 \%$. The eigenfrequency of the pressure tap, the geometry of which is shown in figure 4, was estimated to be approximately $8000 \mathrm{~Hz}$. Blade passage frequencies corresponding to shaft speeds from 1200 to 2400 rpm which were used during testing ranged from 160 to $320 \mathrm{~Hz}$. The spectrum of unsteady pressure measurements (figure 5) taken at suction side tap $2(\mathrm{~S} 2 \mathrm{C})$ at $1800 \mathrm{rpm}$ and $\phi=0.12$ shows that the estimate of the eigenfrequency was reasonable. It can be seen that the blade passages frequency and its higher harmonics are "far" removed from the eigenfrequency of the tap, so that amplification and phase shift of the signal were negligible. All front shroud pressure measurements were made with the transducers flushmounted.

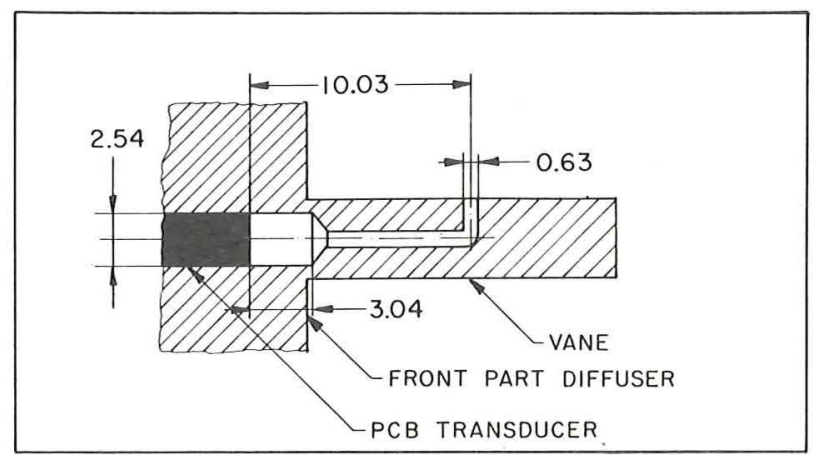

Fig. 4 Geometry of the vane pressure tap.

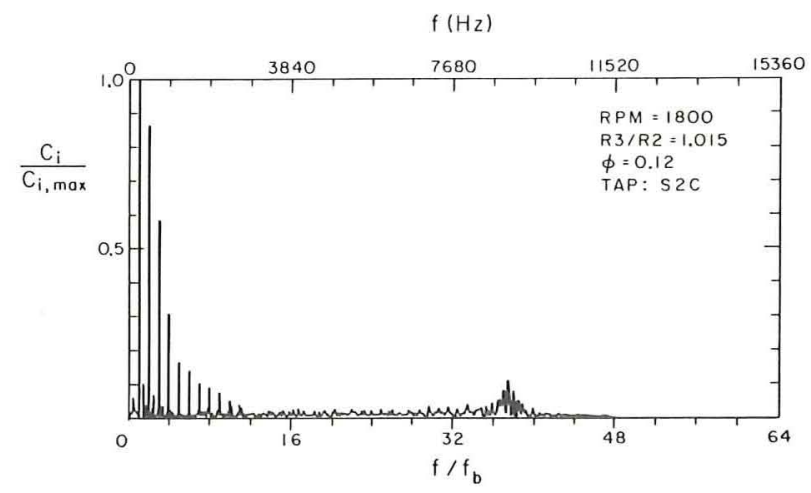

Fig. 5 Spectrum of unsteady vane pressure measurements at pressure tap S2C $\left(\phi=0.12, \mathbf{R}_{3} / \mathbf{R}_{2}=1.015\right)$. 
The data were sampled and discretized in a 16 channel "data taker" made at Caltech. An encoder was used on the main shaft to trigger the data taker and to provide a clock for the data taker. The maximum sampling frequency of the data taker, using one channel for data taking only, is 32 $\mathrm{kHz}$. 1024 data per main shaft cycle were taken for shaft speeds up to $1800 \mathrm{rpm}, 512$ for higher shaft speeds. This corresponds to 128 or 64 data per impeller blade passage, respectively. Since the signal contained some noise, the unsteady data were ensemble averaged over two impeller blade passages, a full and a partial blade passage. For shaft speeds up to 1800 rpm, 2048 full and partial impeller blade passages were used for ensemble averaging, for higher shaft speeds 4096 . The experimental error was found to be less than $\pm 5 \%$ for the magnitude and less than \pm 2 degrees (360 degrees corresponding to one impeller blade passage) for the phase of the ensemble averaged unsteady pressure measurements.

\section{OVERALL PERFORMANCE}

Performance curves for Diffuser $\mathrm{S}$ and Diffuser $\mathrm{T}$ are shown in figure 6 . For the vaned diffuser, the maximum flow coefficient is about $2.5 \%$ and the total head coefficient is up to $9 \%$ larger than for the vaneless diffuser. The design flow coefficient for the impeller was not known. Hence, efficiency measurements were made at a number of shaft speeds to determine the best efficiency flow coefficient for the impeller and Diffuser S. From these results, the best efficiency point was found to be at approximately $\phi=0.12$, nearly independent of shaft speed.

\section{DISCUSSION OF RESULTS}

Data were taken for four flow coefficients, maximum flow coefficient $(\phi=0.15)$, best efficiency flow coefficient $(\phi=0.12)$, and two lower flow coefficients $(\phi=0.09$ and $\phi=0.06)$, at three shaft speeds, 1200,1800 , and $2400 \mathrm{rpm}$, and for two radial gaps , $1.5 \%$ and $4.5 \%$ based on impeller discharge radius, between impeller discharge and the particular instrumented vane. At one tap on both the vane suction and the vane pressure side (S2C and $\mathrm{P} 1 \mathrm{C}$, respectively), measurements were taken for a total of eleven flow coefficients, ranging from $\phi=0.05$ to $\phi=0.15$.

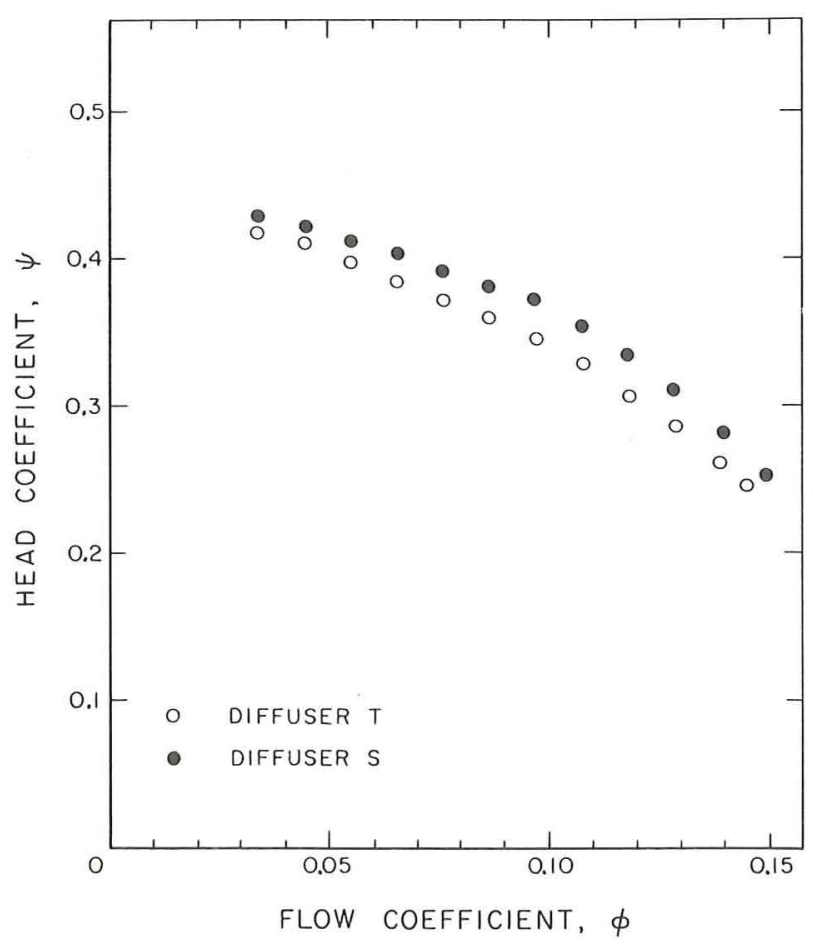

Fig. 6 Performance curves for Diffuser $S$ and Diffuser $T$.

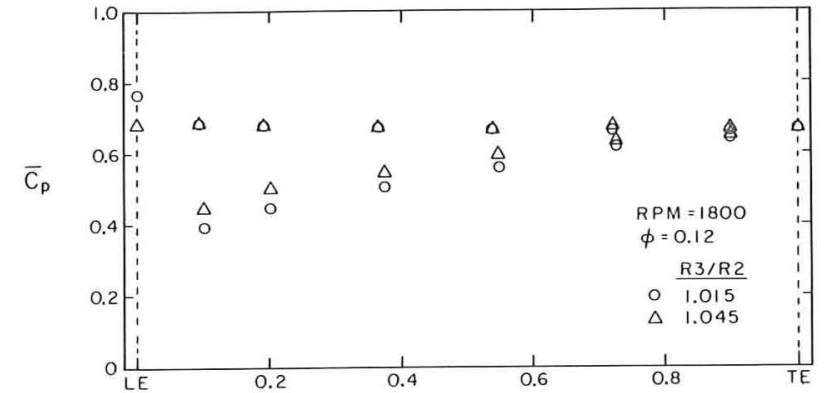

Fig. 7 Steady vane pressure measurements at mid vane height $(\phi=0.12)$.

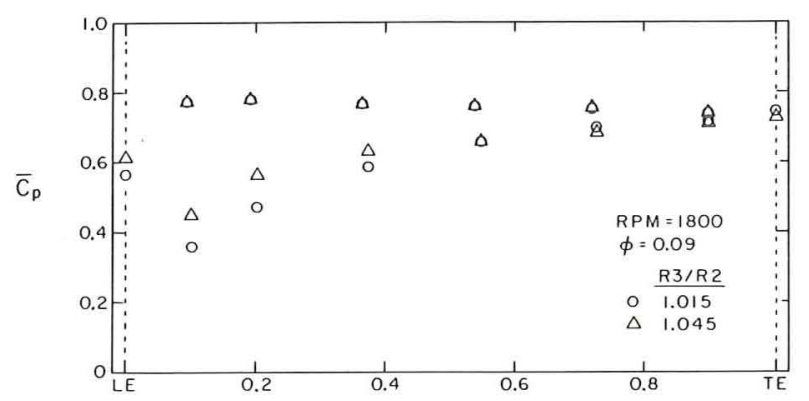

Fig. 8 Steady vane pressure measurements at mid vane height $(\phi=0.09)$.

Steady Vane Pressure Measurements. Normalized by the dynamic pressure based on impeller tip speed, the steady vane pressure measurements will be presented as a steady vane pressure coefficient,

$$
\bar{c}_{p}=\frac{\left(\bar{p}_{v}-\bar{p}_{u p}\right)}{(1 / 2) \rho u_{2}^{2}}
$$

Figures 7 and 8 show the steady vane pressure distribution at mid vane height. The measurements were taken at $1800 \mathrm{rpm}$, for two flow coefficients, $\phi=0.12$ and $\phi=0.09$, and two different radial gaps, $R_{3} / R_{2}=1.015$ and 1.045. The vane loading is larger for $\phi=0.09$ than for $\phi=0.12$. For both flow coefficients, however, the vane pressure increases on the suction side, and remains unchanged on the pressure side with increasing radial gap. Hence, the steady lift on the vane decreases with increasing radial gap. Furthermore, the diffusion on the suction side is clearly noticeable.

Unsteady Vane Pressure Measurements. The unsteady vane pressure measurements are presented as an unsteady vane pressure coefficient, normalized by the dynamic pressure based on impeller tip speed,

$$
\tilde{c}_{p}=\frac{\tilde{p}_{v}}{(1 / 2) \rho u_{2}^{2}}
$$

In figure 9 a sample of unsteady vane pressure measurements taken at pressure tap $\mathrm{S} 2 \mathrm{C}$ for $1800 \mathrm{rpm}$ and $\phi=0.12$ is shown. It is evident that the pressure fluctuations are periodic with impeller blade passage and of the same order of magnitude as the total pressure rise across the pump. The lowest pressure occurs after the impeller blade suction side has passed the diffuser vane leading edge. Again, the noise in the measurement is due to the resonant frequency of the pressure tap.

Spectra of Unsteady Vane Pressure Measurements. In figures 10-14 spectra of unsteady vane pressure measurements made at 1800 rpm are presented. The magnitude of the Fourier coefficients relative to the magnitude of the largest Fourier coefficient $\left(c_{i} / c_{i, \max }\right)$ for a particular test is shown versus frequency (upper horizontal scale) and frequency normal- 


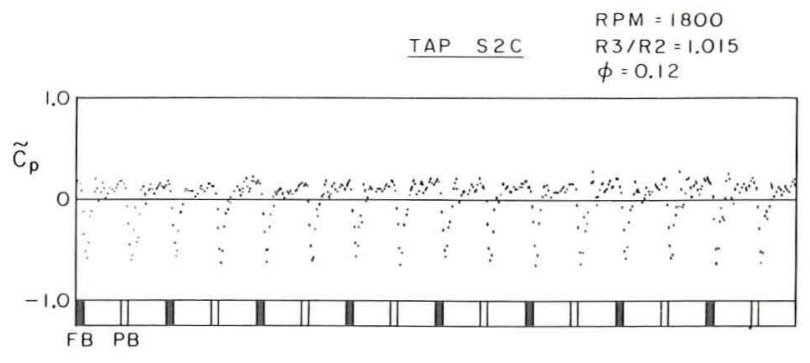

Fig. 9 Unsteady vane pressure measurements at pressure tap S2C.

ized by impeller blade passage frequency $\left(f / f_{b}\right)$ (lower horizontal axis). The magnitude of the largest Fourier coefficient for the particular test is given in the caption of each figure. The spectra were obtained using 4096 data points, corresponding to four shaft revolutions or 32 impeller blade passages (the relative magnitudes of the first 512 frequencies are shown).

The spectrum presented in figure 10 corresponds to the unsteady data presented in figure 9, taken at best efficiency flow coefficient, $\phi=0.12, \mathrm{rpm}$ $=1800$, and $R_{3} / R_{2}=1.015$. The dominant frequencies are the impeller blade passage frequency and its higher harmonics, which decay very slowly. At $f / f_{b}=1 / 2,3 / 2,5 / 2$ etc., the relative magnitudes of the Fourier coefficients corresponding to full impeller blade passage frequency, $f_{f b}$, and its higher harmonics can be seen (since the impeller has a total of eight blades, four full and four partial blades, the full impeller blade passage frequency, $f_{f b}$, is equal to the partial impeller blade passage frequency, $f_{p b}$, and furthermore, $\left.f_{f b}=(1 / 2) f_{b}\right)$. The relative magnitudes of the Fourier coefficients at those frequencies indicate the difference on the vane pressure measurements between a full and a partial impeller blade passing the diffuser vane. For the measurements presented in figure 10, those differences are small. In figure 11, the spectrum of unsteady measurements at the same pressure tap and for the same flow coefficient but with the radial gap increased from $1.5 \%$ to $4.5 \%$ is presented. The relative magnitudes of the higher blade passage harmonics are smaller for the increased radial gap. The magnitude of the largest Fourier coefficient, at impeller blade passages frequency, is reduced by about $50 \%$ with the radial gap increased from $1.5 \%$ to $4.5 \%$. Figure 12 shows the spectrum of unsteady measurements taken near the vane trailing edge on the suction side ( $\operatorname{tap} \mathrm{S} 6 \mathrm{C}$ ) for $\phi=0.12$ and a radial gap of $1.5 \%$. The blade passage frequency is dominant; however, its magnitude has decreased to about a third of the magnitude of the impeller blade passage frequency at tap S2C, and its higher harmonics decay much faster than at tap S2C (figure 10). But, even on the rear part of the vane the fluctuations are still periodic with impeller blade passage frequency.

At some pressure taps, the relative magnitude of the full impeller blade passage frequency depends strongly on flow coefficient. This is shown for pressure tap P1C in figures 13 and 14 . Decreasing the flow coefficient from $\phi=0.12$ to $\phi=0.08$ results in a significant increase in the relative magnitude of the full blade passage frequency, from less than 0.1 to about 0.55 . Thus, for $\phi=0.08$ at pressure tap P1C, there is a significant difference in the pressure fluctuations caused by a full or a partial impeller blade passing by the diffuser vane.

Magnitudes of Vane Pressure Fluctuations. In figures 15-17 data on the magnitude of the ensemble averaged unsteady vane pressure fluctuations at mid vane height are presented. The fluctuations are defined as the difference between the maximum and minimum pressure value in the averaging period which corresponds to one full and one partial impeller blade passage. It can be seen that the largest fluctuations independent of experimental parameters such as flow coefficient, shaft speed or radial gap occur on the front half of the suction side of the vane. Those fluctuations are of the same order of magnitude as the total pressure gain across the pump. Furthermore, the fluctuations on the pressure side are significantly smaller than those on the suction side. It is interesting to notice, that although the fluctuations decrease on both pressure and suction side along the vane, they attain a relative maximum on the rear half of the vane. This observation is similar to that made by Dring [1] in turbine rotor-stator interaction. Dring also reported larger blade pressure fluctuations on the turbine rotor suction side than on the pressure side. In the compressor stage investigated by Gallus [2], the largest fluctuations on the compressor stator were reported to

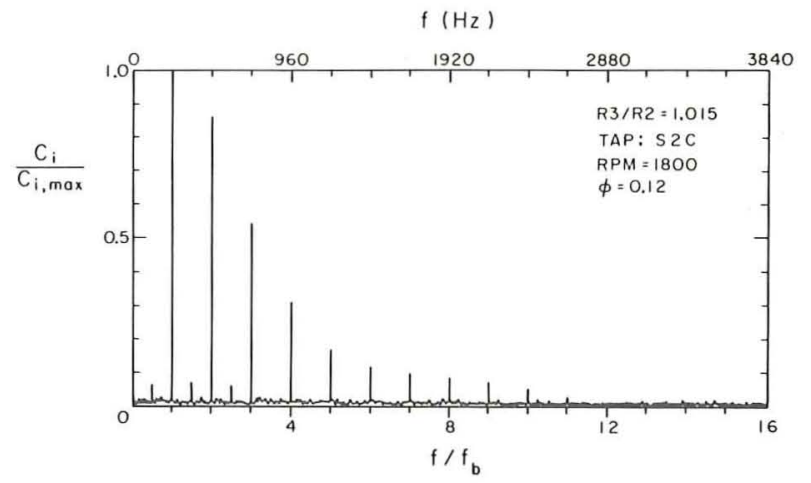

Fig. 10 Spectrum of unsteady vane pressure measurements at pressure tap $\mathrm{S} 2 \mathrm{C}\left(\phi=0.12, \mathbf{R}_{8} / \mathbf{R}_{2}=1.015, \mathrm{c}_{\mathrm{i}, \max }=\right.$ $0.194)$.

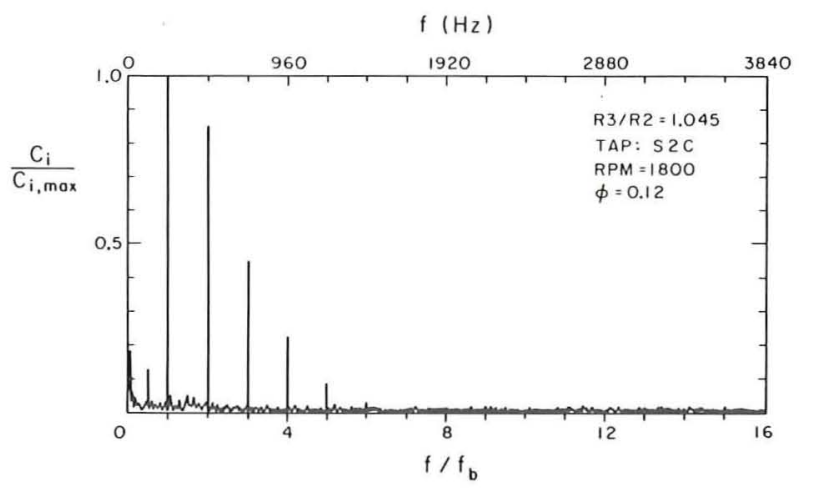

Fig. 11 Spectrum of unsteady vane pressure measurements at pressure tap S2C $\left(\phi=0.12, R_{8} / R_{2}=1.0 \$ 5, c_{1, \max }=\right.$ 0.096).

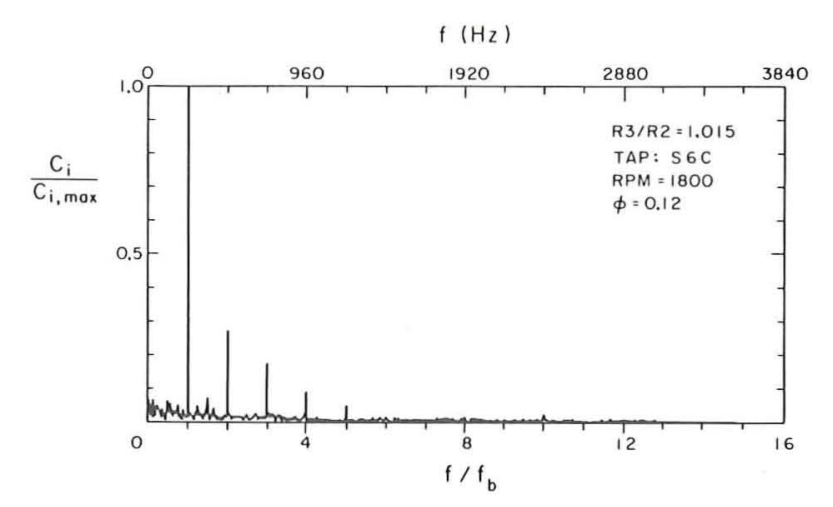

Fig. 12 Spectrum of unsteady vane pressure measurements at pressure tap S6C $\left(\phi=0.12, \mathbf{R}_{3} / \mathbf{R}_{\mathbf{2}}=1.015, \mathrm{c}_{\mathrm{i}, \max }=\right.$ $0.070)$.

occur at a tap on the pressure side. However, taking into account all the measurements reported on both pressure and suction side, it appears that the mean magnitude of the fluctuations is larger at the suction side taps than at the pressure side taps. 


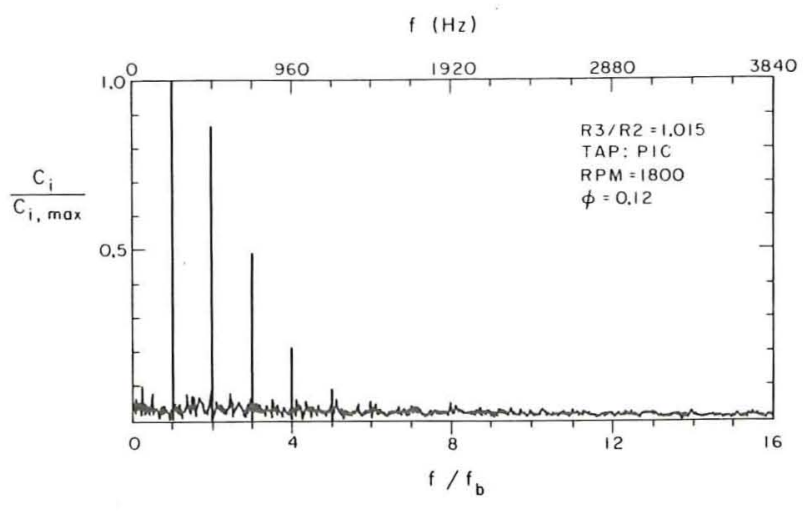

Fig. 13 Spectrum of unsteady vane pressure measurements at pressure tap P1C $\left(\phi=0.12, \mathbf{R}_{3} / \mathbf{R}_{2}=1.015, \mathrm{c}_{1, \max }=\right.$ $0.060)$.

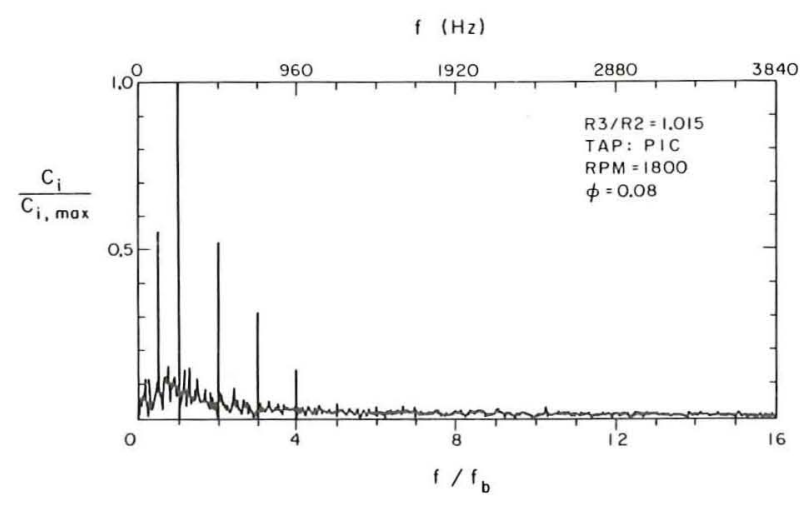

Fig. 14 Spectrum of unsteady vane pressure measurements at pressure tap P1C $\left(\phi=0.08, \mathbf{R}_{3} / \mathbf{R}_{2}=1.015, c_{i, \max }=\right.$ $0.071)$.

Figure 15 shows the dependence of the fluctuations on the radial gap for $\phi=0.12$. Increasing the radial gap resulted in a significant decrease, of about $50 \%$, of the large fluctuations on the front half of the vane suction side and at the pressure tap on the pressure side closest to the leading edge. At most other measurement locations the fluctuation decreased slightly or remained constant. Only at one pressure tap, P5C, on the rear half of the pressure side, did they increase with increasing radial gap. Figure 16 compares the magnitude of the fluctuations at different shaft speeds, where data are presented for $\phi=0.12$ and a radial gap of $1.5 \%$. With the exception of the pressure tap at the vane trailing edge, there is hardly any speed dependence of the normalized pressure fluctuations at any of the pressure taps. In figure 17 the magnitude of the fluctuations for four different flow coefficients, $\phi=0.15,0.12,0.09$, and 0.06 at $1800 \mathrm{rpm}$ and a radial gap of $4.5 \%$ is shown. At all pressure taps on the suction side, the fluctuations are largest for maximum flow, $\phi=0.15$. At two suction side taps(S1C and $\mathrm{S} 5 \mathrm{C})$ the fluctuations decrease significantly with decreasing flow coefficient, whereas at all other suction side taps they are not significantly different for $\phi=0.12, \phi=0.09$, and $\phi=0.06$. On the vane pressure side, where the fluctuations are significantly smaller than one the suction side, the differences in magnitude of the fluctuations for the four flow coefficients investigated are small at most pressure taps, except at P1C, where the fluctuations are largest for $\phi=0.09$ and for P2C, where they are largest for $\phi=0.15$.

To investigate in more detail the dependence of the magnitude of the fluctuations on the flow coefficient, measurements were made for radial gaps of $1.5 \%$ and $4.5 \%$ for a total of eleven flow coefficients, ranging from $\phi=$ 0.05 to $\phi=0.15$, on two pressure taps, P1C and S2C. The magnitude of the fluctuations as functions of flow coefficient and radial gap, relative

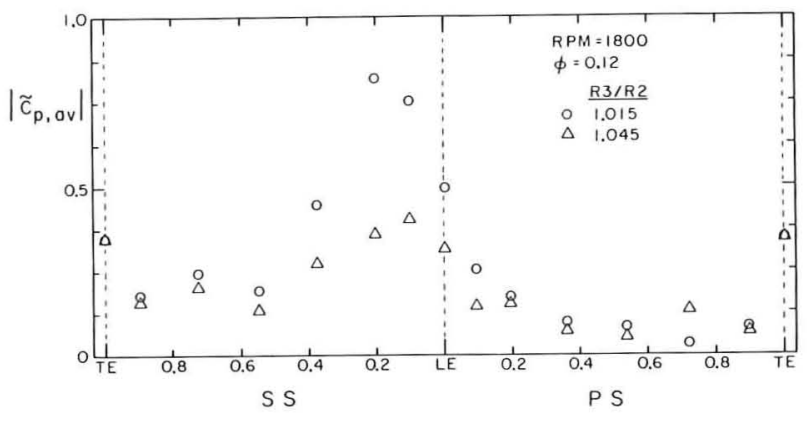

Fig. 15 Magnitude of ensemble averaged pressure fluctuations at mid vane height $\left(\phi=0.12, R_{3} / R_{2}=1.015\right.$ and 1.045$)$.

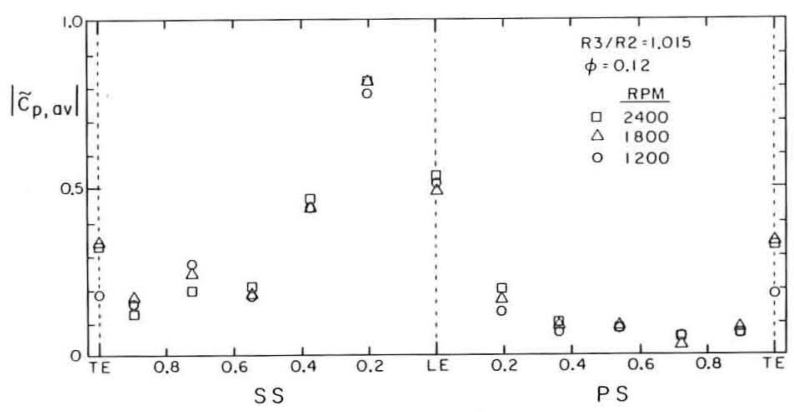

Fig. 16 Magnitude of ensemble averaged pressure fluctuations at mid vane height $(\phi=0.12, \mathrm{RPM}=$ $1200,1800$ and 2400$)$.

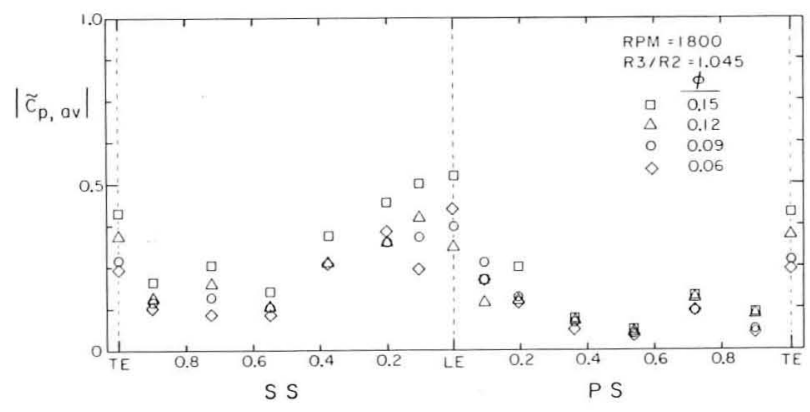

Fig. 17 Magnitude of ensemble averaged pressure fluctuations at mid vane height $\left(\mathbf{R}_{3} / \mathbf{R}_{3}=1.045, \phi=\right.$ $0.15,0.12,0.09$ and 0.06$)$.

to the magnitude of the fluctuation for maximum flow $(\phi=0.15)$ at a radial gap of $1.5 \%$ is presented for tap P1C in figure 18 and for tap $\mathrm{S} 2 \mathrm{C}$ in figure 19. For both taps, the fluctuations are largest for maximum flow at a radial gap of $1.5 \%$; however, they show different behavior for decreasing flow coefficient. For P1C at a radial gap of $1.5 \%$, they attain a minimum for $\phi=0.11$, dropping about $40 \%$ below the maximum fluctuations for $\phi=0.15$. Increasing the radial gap to $4.5 \%$ results in a shift of the minimum fluctuations from $\phi=0.11$ to $\phi=0.12$. Furthermore, the largest fluctuations no longer occur for maximum flow, but for $\phi=0.09$. For tap S2C, the fluctuations are largest, for both radial gaps, for-maximum flow, $\phi=0.15$, and decrease with decreasing flow coefficient. They attain relative minima and maxima for $\phi=0.09$ and $\phi=0.07$ (for a radial gap of $1.5 \%$ ), and for $\phi=0.11$ and $\phi=0.07$ (for a radial gap of $4.5 \%$ ). 
Furthermore, the relative decrease of the pressure fluctuations with increasing radial gap is significantly different for the two taps. For tap P1C, the fluctuations decrease most strongly, by about $50 \%$, for maximum flow, $\phi=0.15$, and for the smallest flow coefficient investigated, $\phi=0.05$, when increasing the radial gap from $1.5 \%$ to $4.5 \%$. However, for medium flow coefficients such as $\phi=0.10$ the fluctuations decrease only by about $10 \%$. For pressure tap $\mathrm{S} 1 \mathrm{C}$, the pressure fluctuations decrease with increasing radial gap most strongly, by about $60 \%$, for maximum flow, $\phi=0.15$. Lowering the flow coefficient, the decrease of the fluctuations with increasing radial gap is reduced slightly, up to about $50 \%$ for $\phi=0.05$.

Pressure measurements were also made on the suction side at four axial positions across the vane at two distances downstream of the leading edge ( $\mathrm{s}=0.10$ and 0.37 ; the two pressure taps at mid vane height corresponding to $\mathrm{s}=0.10$ and $\mathrm{s}=0.37$ are $\mathrm{S} 1 \mathrm{C}$ and $\mathrm{S} 3 \mathrm{C}$, respectively). In figure 20 the magnitude of the ensemble averaged fluctuations are presented. At $s=0.10$, the fluctuations near the hub were found to be more than twice as large than those near the shroud. At $s=0.37$, the fluctuations near the hub were still found to be larger than those near the shroud; but, the differences, especially for a radial gap of $4.5 \%$, were smaller than at $\mathrm{s}=0.10$.

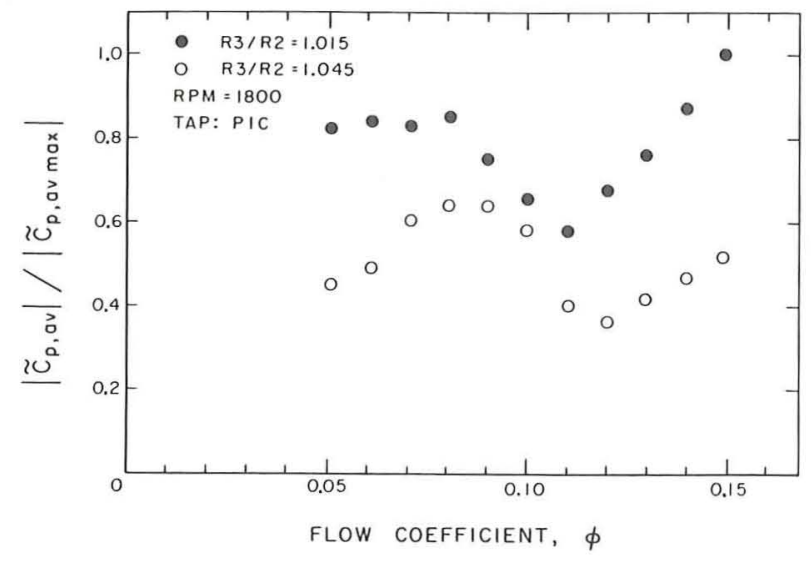

Fig. 18 Magnitude of ensemble averaged pressure fluctuations at pressure tap $\mathrm{P} 1 \mathrm{C}\left(\mathbf{R}_{\mathbf{3}} / \mathbf{R}_{\mathbf{2}}=1.015\right.$ and $1.045, \phi=$ $0.05-0.15)$. Best efficiency flow coefficient: $\phi=0.12$.

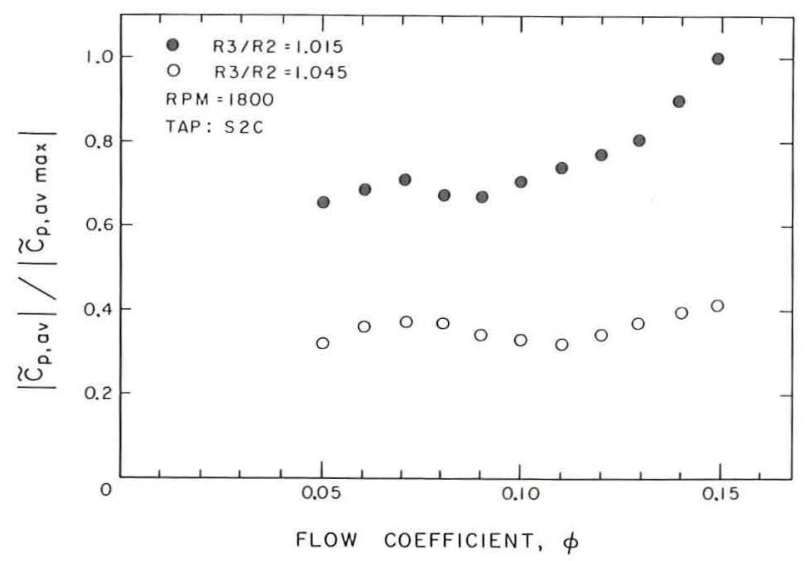

Fig. 19 Magnitude of ensemble averaged pressure fluctuations at pressure tap $\mathrm{S} 2 \mathrm{C}\left(\mathbf{R}_{3} / \mathbf{R}_{2}=1.015\right.$ and $1.045, \phi=$ $0.05-0.15)$. Best efficiency flow coefficient: $\phi=0.12$.
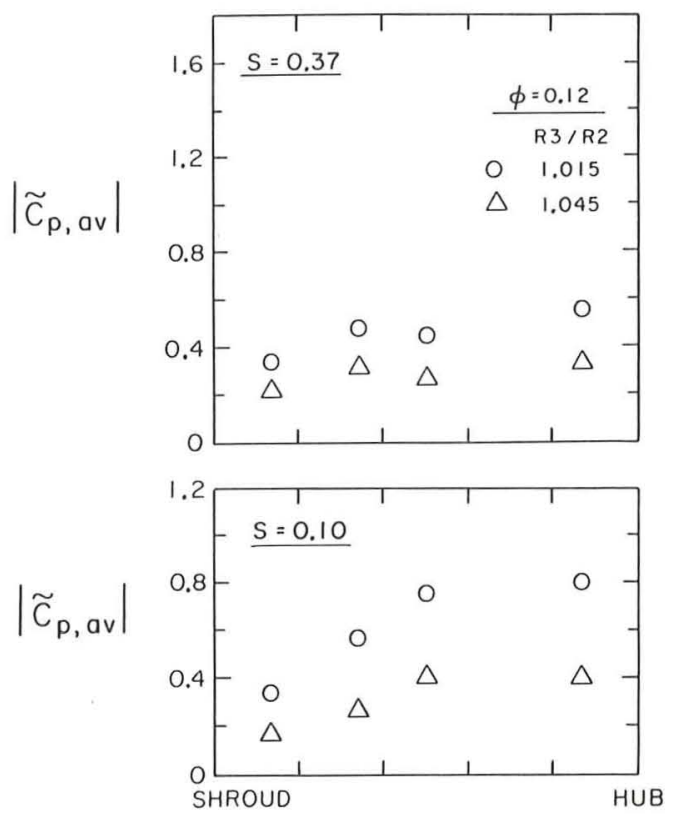

Fig. 20 Magnitude of ensemble averaged pressure fluctuations across the span of the vane $\left(\phi=.12, \mathbf{R}_{\mathbf{3}} / \mathbf{R}_{\mathbf{2}}=\right.$ 1.015 and 1.045).

Steady and Unsteady Lift Computations. From the vane pressure measurements described earlier, the force on the vane at mid vane height was computed. Since those measurements were obtained on different vanes they had to be phase shifted to one reference vane for the force computations. The steady force was computed from the steady pressure distribution around the vane. Superimposing the steady and ensemble averaged unsteady pressure measurements, the ensemble averaged vane pressure distribution was obtained (it was assumed that the steady pressure value obtained by the mercury manometer measurements was identical to the time mean value about which the piezoelectric transducer measured the unsteady pressure). From the ensemble averaged pressure distribution, the ensemble averaged force was computed. The steady and the ensemble averaged pressure distribution on the vane was obtained by fitting a third order periodic spline through the measured pressure values. A periodic spline fit was chosen to get continuity for the pressure and the first two pressure derivatives at the leading and trailing edge. Normalized, the steady and ensemble averaged force were computed from the equations,

$$
\begin{gathered}
\overline{\mathbf{F}}=-\oint \frac{\left(\bar{p}_{v}-\bar{p}_{u p}\right)(\xi)}{\rho u_{2}^{2} c} \mathbf{n} d \xi \\
\mathbf{F}_{\mathbf{a v}}=-\oint \frac{\left(\bar{p}_{v}+\tilde{p}_{v, a v}-\bar{p}_{u p}\right)(\xi)}{\rho u_{2}^{2} c} \mathbf{n} d \xi
\end{gathered}
$$

The lift on the vane was defined as the component of the force on the vane normal to the chord joining the vane leading and trailing edge. The lift on the vane was defined positive if the force component normal to the vane chord is in positive y direction (figure 3a).

In figure 21 the ensemble averaged vane pressure distribution at mid vane height is shown for two different locations of the impeller blades relative to the instrumented vane for best efficiency flow coefficient, $\phi=0.12$, at $1800 \mathrm{rpm}$, and a radial gap of $1.5 \%$. (The full circles indicate actual measurement points, the curve connecting those points the pressure distribution as obtained by the interpolation scheme). As the impeller blade passes the diffuser vane, the vane pressure on the vane suction side drops below upstream pressure, resulting in a large pressure difference between vane suction and pressure side. When the impeller blade is approximately halfway between two diffuser vanes the vane pressure distribution is similar to the steady pressure distribution presented in figure 7 , except for the pressure fluctuations at the trailing edge. 

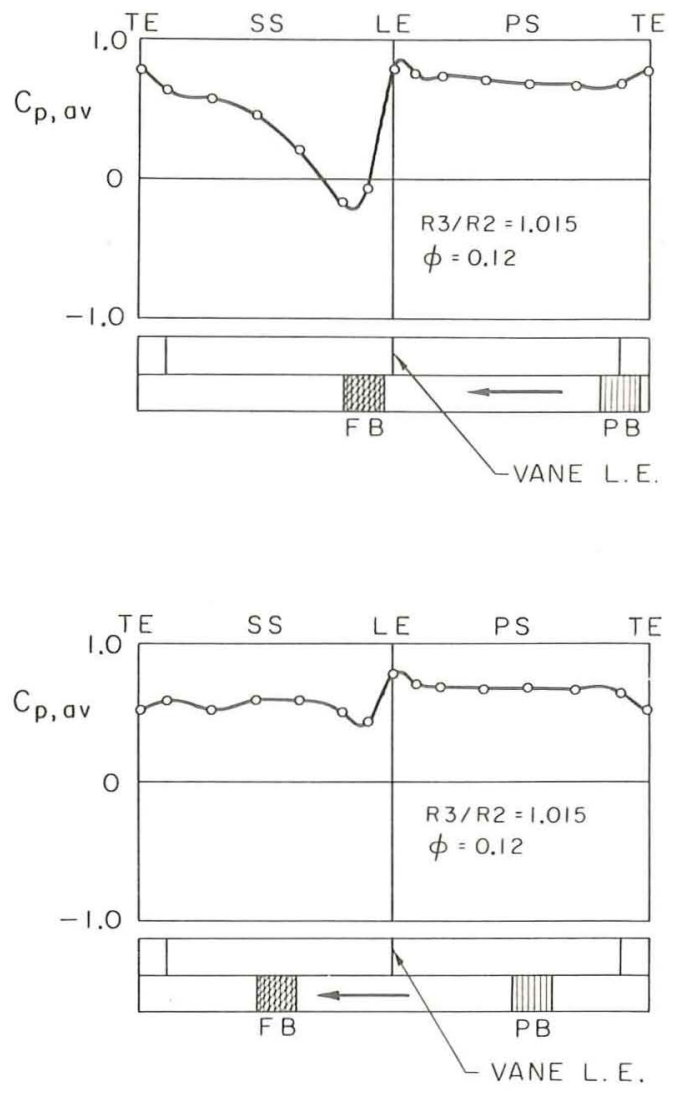

Fig. 21 Ensemble averaged pressure distribution at mid vane height $\left(\phi=0.12, \mathbf{R}_{3} / \mathbf{R}_{2}=1.015\right)$.

In figure 22 the lift on a diffuser vane is presented for $\phi=0.12$, $\mathrm{rpm}=1800$, and radial gaps of $1.5 \%$ and $4.5 \%$. The position of the impeller blades (shown is a full and a partial blade) is referenced to the diffuser vane leading edge of the instrumented vane. Maximum lift is attained immediately after the impeller blade suction side has passed the vane leading edge. As seen in figure 20 , for a radial gap of $1.5 \%$ the vane pressure on the suction side in the vicinity of the leading edge drops below upstream pressure at this instant of time. As the impeller blade moves on, the lift decreases, until the blade pressure side passes the vane leading edge. At this instant, the lift on the vane for the radial gap of $1.5 \%$ is slightly negative. Then, as the impeller blade trailing edge passes by, the lift increases sharply from its minimum to its maximum value. The increase of the radial gap from $1.5 \%$ to $4.5 \%$ results in a significant decrease in the fluctuating lift, as expected from a similar decrease of the magnitude of the vane pressure fluctuations.

The ratio of ensemble averaged lift to steady lift is presented in figure 23 . It was found that the magnitude of the ensemble averaged lift was up to three times larger than the steady lift. Furthermore, the ratio of ensemble averaged lift to steady lift decreased with increasing radial gap, which was not obvious a priori since an increase in radial gap resulted in a decrease in both the steady lift, figure 7, and the magnitude of the pressure fluctuations, figure 15.

In figure 24 the ensemble averaged lift on the vane is presented for $\phi=0.15,0.12$, and 0.09 for a radial gap $4.5 \%$. The fluctuations are largest for $\phi=0.15$ and decrease for $\phi=0.12$, and $\phi=0.09$. Differences in the magnitude of the lift fluctuations depending upon a partial or a full impeller blade passing the diffuser vane were observed. For best efficiency point and just below $(\phi=0.12$ and $\phi=0.09)$, the lift fluctuations were larger for a full impeller blade passing the vane than for a partial impeller blade (by $5 \%$ and $22 \%$, respectively). For maximum flow $(\phi=0.15)$, however, the lift fluctuations were by about $8 \%$ larger for a partial impeller blade passing the vane than for a full impeller blade. The large difference in lift fluctuation between a full and a partial impeller blade passing the diffuser vane for the low flow coefficient, $\phi=0.09$, coincides with the results of the spectra presented earlier. The spectra showed that with decreasing flow coefficient, from $\phi=0.12$ to $\phi=0.08$, the relative magnitude of the full blade passage frequency may increase significantly. The observations that the fluctuating lift decreases with decreasing flow coefficient is different from that reported by Gallus [2],[3] where the fluctuating lift on the stator blades was smallest for maximum flow, and increased with decreasing flow coefficient.

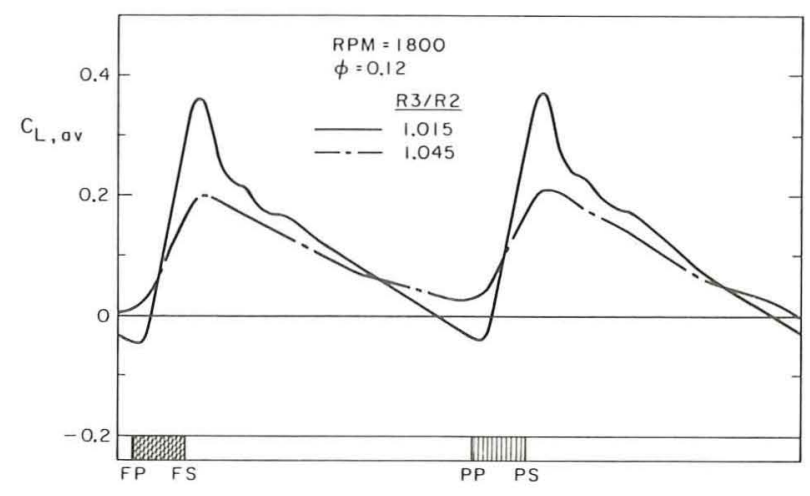

Fig. 22 Ensemble averaged lift on diffuser vane at mid vane height $\left(\phi=0.12, R_{8} / R_{2}=1.015\right.$ and 1.045$)$.

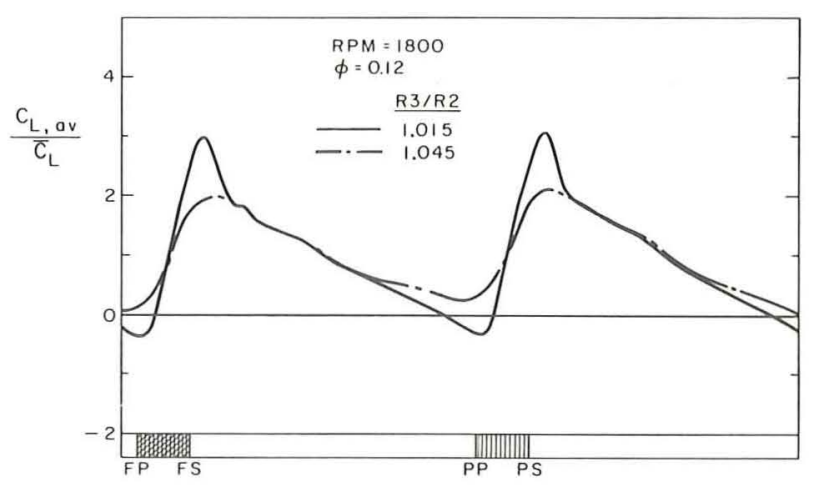

Fig. 23 Ratio of ensemble averaged lift to steady lift at mid vane height $\left(\phi=0.12, \mathbf{R}_{3} / \mathbf{R}_{2}=1.015\right.$ and 1.045$)$.

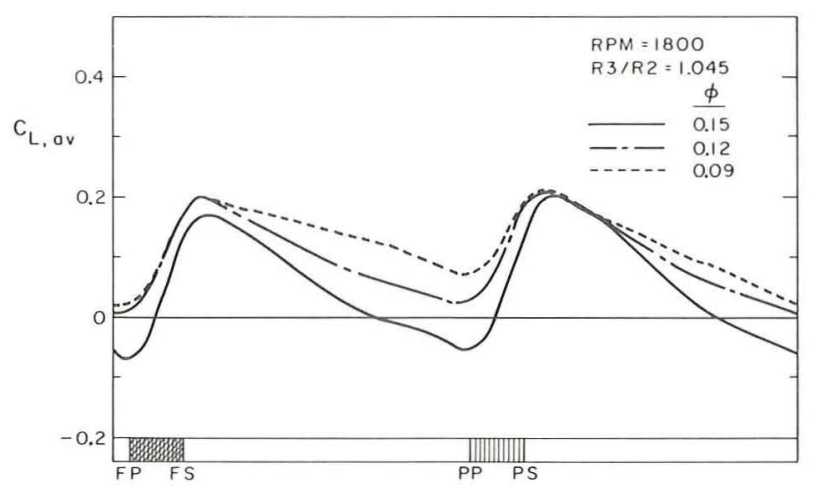

Fig. 24 Ensemble averaged lift on diffuser vane at mid vane height $\left(\phi=0.15,0.12\right.$ and $\left.0.09, \mathbf{R}_{3} / \mathbf{R}_{2}=1.045\right)$. 


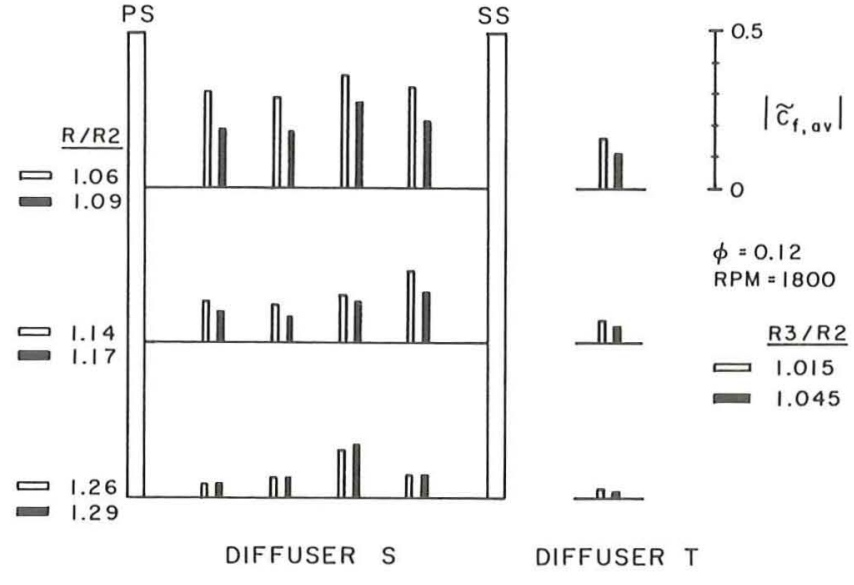

Fig. 25 Magnitude of ensemble averaged front shroud pressure fluctuations on Diffuser $\mathbf{S}$ and Diffuser $\mathbf{T}(\phi=$ $0.12, R_{8} / R_{2}=1.015$ and 1.045$)$.

Front Shroud Pressure Measurements. Unsteady pressures measurements were made on the front shroud of the vaned diffuser(Diffuser S), and a second diffuser of identical geometry but vaneless (Diffuser T). For Diffuser $\mathrm{T}$, the measurements were made at three distances to the diffuser inlet; for Diffuser $\mathrm{S}$ at any of those three distances to the diffuser inlet four measurements were made between the pressure and the suction side of two adjacent diffuser vanes (figure $3 \mathrm{~b}$ ). In figure 25 data on the magnitude of the ensemble averaged front shroud pressure fluctuations are presented for $\phi=0.12$ and radial gaps of $1.5 \%$ and $4.5 \%$ between impeller discharge and the diffuser inlet. The ratio $R / R_{2}$ in figure 25 describes the ratio of the radius of the particular front shroud pressure measurement location to the impeller discharge radius for the different radial gaps between impeller discharge and diffuser inlet (for Diffuser $\mathrm{S}$ the radial gaps between impeller discharge and diffuser vane leading edge and between impeller discharge and diffuser inlet are identical). It can be noticed, that the fluctuations on the front shroud of the vaned diffuser are about twice as large as those on the vaneless diffuser. This is a clear indication that for the range of radial gaps investigated in this experiment both the potential interaction between impeller blades and diffuser vanes and the convection of the wakes shed by the impeller blades contribute to the unsteadiness of the flow. Furthermore, for the vaned diffuser, the magnitude of the front shroud pressure fluctuations depends on the circumferential position between suction and pressure side of two adjacent vanes. The fluctuations seem to be smaller and to decay faster in the vicinity of the pressure side than the vicinity of the suction side.

\section{CONCLUSION}

Steady and unsteady vane and front shroud pressure measurements were made for one half of the double suction pump impeller of the HPOTP (High Pressure Oxygen Turbopump) of the SSME (Space Shuttle Main Engine) and a vaned and a vaneless diffuser to investigate rotor-stator interaction in diffuser pumps. Spectra of the unsteady vane pressure measurements showed that the pressure fluctuations were periodic with impeller blade passage frequency. The vane pressure fluctuations were found to be larger on the suction side than on the pressure side attaining their maximum value, of about the same order of magnitude as the total pressure rise across the pump, in the vicinity of the leading edge. For a radial gap of $1.5 \%$ (based on impeller discharge radius) between impeller discharge and diffuser vane leading edge, the pressure on the vane suction side was observed to drop locally below upstream pressure immediately after the impeller blade trailing edge has passed the vane leading edge. The large pressure fluctuations on the front half of the vane suction side decreased by about $50 \%$ when the radial gap was increased from $1.5 \%$ to $4.5 \%$. The lift on the vane, steady and unsteady, was computed from the vane pressure measurements. The magnitude of the fluctuating lift was found to be larger than the steady lift thus subjecting the vane to a large periodic load. Fluctuating lift as well as ratio of lift to steady lift decreased strongly when the radial gap was increased from $1.5 \%$ to $4.5 \%$. Unsteady pressure measurements at different locations across the span of the vane showed that the pressure fluctuations near the hub were considerably larger than those near the shroud. The unsteady front shroud pressure fluctuations on the vaned diffuser were found to be considerably larger than those on a vaneless diffuser, indicating that the close spacing of impeller and diffuser strongly increased the unsteadiness of the flow.

\section{ACKNOWLEDGEMENTS}

The authors are indebted to the NASA George Marshall Space Flight Center for continued sponsorship of this research under contract NAS833108.

\section{REFERENCES}

1 Dring,R.P., Joslyn,H.D., Hardin,L.W., and Wagner,J.H., "Turbine Rotor-Stator Interaction," ASME Journal of Engineering for Power, Oct. 1982, Vol.104,pp. 729-742.

2 Gallus,H.E., "High Speed Blade-Wake Interactions," von Kármán Institute for Fluid Mechanics Lecture Series 1979-3, Jan. 29-Feb. 2 1979, Volume 2.

3 Gallus,H.E., Lambertz,J., and Wallmann,T., "Blade-Row Interaction in an Axial Flow Subsonic Compressor Stage," ASME Journal of Engineering for Power, Jan 1980,Vol.102,pp. 169-177.

4 Iino,T., and Kasai,K., "An Analysis of Unsteady Flow Induced by Interaction Between a Centrifugal Impeller and a Vaned Diffuser," (in Japanese), Transactions of the Japan Society of Mechanical Engineers, 1985, Vol. 51, No. 471, pp. 154-159.

5 Krain,H., "A Study on Centrifugal Impeller and Diffuser Flow," ASME Journal of Engineering for Power, Oct. 1981, Vol. 103, pp. 688-697.

6 Inoue,M., and Cumptsy,N.A., "Experimental Study of Centrifugal Impeller Discharge Flow in Vaneless and Vaned Diffusers," ASME Journal of Engineering for Gas Turbines \& Power, April 1984, Vol. 106, pp. 455-467.

7 Arndt,N., Acosta,A.J., Brennen,C.E., and Caughey,T.K., "Unsteady Diffuser Vane Pressure and Impeller Wake Measurements in a Centrifugal Pump," Conference Proceedings 8. Conference on Turbomachinery, Budapest Sept. 1987. 